\title{
Extraventricular neurocytoma in the left temporal lobe: A case report and review of the literature
}

\author{
YU-CHEN JI $^{1,2^{*}}$, JING-XIA HU ${ }^{1,2^{*}}$, YAN LI $^{2}$, PENG-XIANG YAN ${ }^{3}$ and HUAN-CONG ZUO ${ }^{1,2}$ \\ ${ }^{1}$ Department of Neurosurgery, Tsinghua University Yuquan Hospital, \\ Beijing 100040; ${ }^{2}$ Medical Center, Tsinghua University, Beijing 100084; ${ }^{3}$ Department of Neurosurgery, \\ Beijing Tiantan Hospital, Capital Medical University, Beijing 100050, P.R. China
}

Received February 5, 2015; Accepted February 19, 2016

DOI: $10.3892 / \mathrm{ol} .2016 .4487$

\begin{abstract}
Central neurocytoma (CNC) often develops in the ventricular system adjacent to the interventricular foramen and septum pellucidum. According to the World Health Organization, CNCs are classified as grade II tumors, and in recent years it has been reported that CNCs have occasionally occurred in rare areas of the central nervous system. The current study describes a rare case of CNC located in the left temporal lobe of a 49-year-old man, who had been experiencing headaches for 3 weeks. Computed tomography identified a round, well-demarcated, $3.3-\mathrm{cm}$ tumor in the left temporal lobe. The patient underwent surgery and the tumor was totally resected. Histological analysis demonstrated that the resected tumor tissue contained clusters of small cells with regular nuclear morphology, and round nuclei with fine chromatin. Immunohistochemically, neuronal differentiation markers, including synaptophysin and neuronal nuclear antigen, were expressed in the tumor cells. Histopathological examination of the resected tissue confirmed a diagnosis of extraventricular neurocytoma. Magnetic resonance imaging was performed at 3 months post-surgery and demonstrated no evidence of tumor recurrence.
\end{abstract}

\section{Introduction}

Central neurocytomas (CNCs) are rare brain tumors that originate from neuroepithelial tissue, occurring most frequently

Correspondence to: Professor Peng-Xiang Yan, Department of Neurosurgery, Beijing Tiantan Hospital, Capital Medical University, 6 Tiantan Xili, Beijing 100050, P.R. China

E-mail: yanneurosurgeon@126.com

Professor Huan-Cong Zuo, Department of Neurosurgery, Tsinghua University Yuquan Hospital, 5 Shijingshan Road, Shijingshan, Beijing 100040, P.R. China

E-mail: zuoneurosurgeon@126.com

${ }^{*}$ Contributed equally

Key words: neurocy toma, extraventricular, literature review in young adults, and are often located in the lateral ventricles. CNC was first described by Hassoun et al (1) in 1982, and accounts for $0.1-0.5 \%$ of all primary brain tumors (2). Complete surgical resection is currently the optimal treatment choice, and the prognosis of CNC is often favorable due to its benign nature (3). Although CNC typically occurs in the lateral ventricles, a few cases of $\mathrm{CNC}$ occurring in extraventricular regions have been reported in the literature.

\section{Case report}

In March 2014, a 49-year-old man was admitted to the Tsinghua University Yuquan Hospital (Beijing, China) after experiencing headaches for 3 weeks. No other focal neurological deficits were observed upon admission. Computed tomography (CT; SOMATOM Sensation 16; Siemens AG, Munich, Germany) of the brain identified an isodense, round, well-demarcated, $3.3-\mathrm{cm}$ tumor, which was located in the left temporal lobe, in addition to peritumoral edema (Fig. 1A). Marked homogeneous enhancement was observed within the mass on T1-gadolinium imaging (Fig. 1B). The patient underwent surgery, and the tumor was excised via a left temporal craniotomy. As there was no adhesion between the tumor and the dura or the brain, the radical resection of the lesion was straightforward. The pathological report stated that the tumor was a grade II extraventricular neurocytoma (EVN) according to the World Health Organization (WHO) classification of tumors of the central nervous system (4). Resected tissue specimens were formalin (Yuekai Trading Co., Ltd., Hebei, China)-fixed, paraffin (Sinopharm Chemical Reagent Co., Ltd., Shanghai, China)-embedded, cut into $8-\mu \mathrm{m}$ sections, stained with hematoxylin and eosin (Shanghai Qianchen Biotechnologies, Co., Ltd., Shanghai, China) and visualized using an optical microscope (CX23; Olympus Corporation, Tokyo, Japan). Histologically, the resected tumor tissue contained clusters of small cells with regular nuclear morphology, and round nuclei with fine chromatin (Fig. 2). Vascular hyperplasia was also observed. There was no evidence of ischemic necrosis or hemorrhage. For immunohistochemical staining, tissue sections were incubated with monoclonal mouse anti-rat synaptophysin (cat. no. ab11105; 1:500; Abcam, Cambridge, MA, USA), rabbit anti-mouse neuronal nuclear antigen (cat. no. ab190565; 1:50; Abcam) 

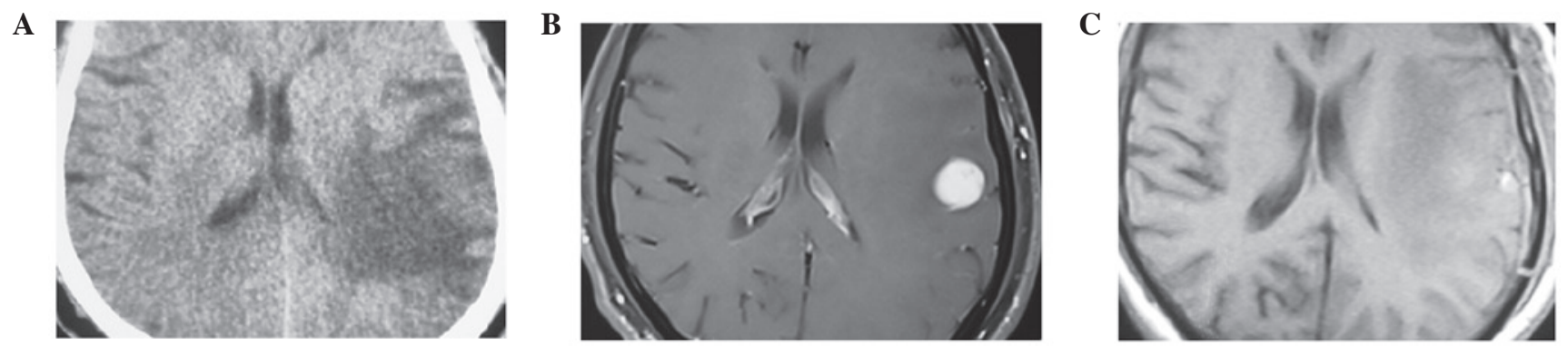

Figure 1. Pre- and post-surgery brain scans of the present case. (A) CT revealing a isodense, well-demarcated, round tumor located in the left temporal lobe, in addition to peritumoral edema. (B) T1-gadolinium image revealing a homogeneously-enhanced mass. (C) Post-operative magnetic resonance imaging demonstrating no signs of solid tumor recurrence. CT, computed tomography.

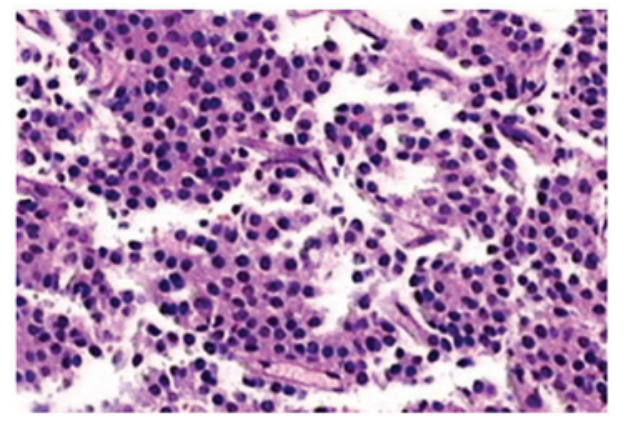

Figure 2. Histological analysis of the resected tissue exhibiting clusters of small cells with regular nuclear morphology and round nuclei. Staining, hematoxylin and eosin; magnification, x100.

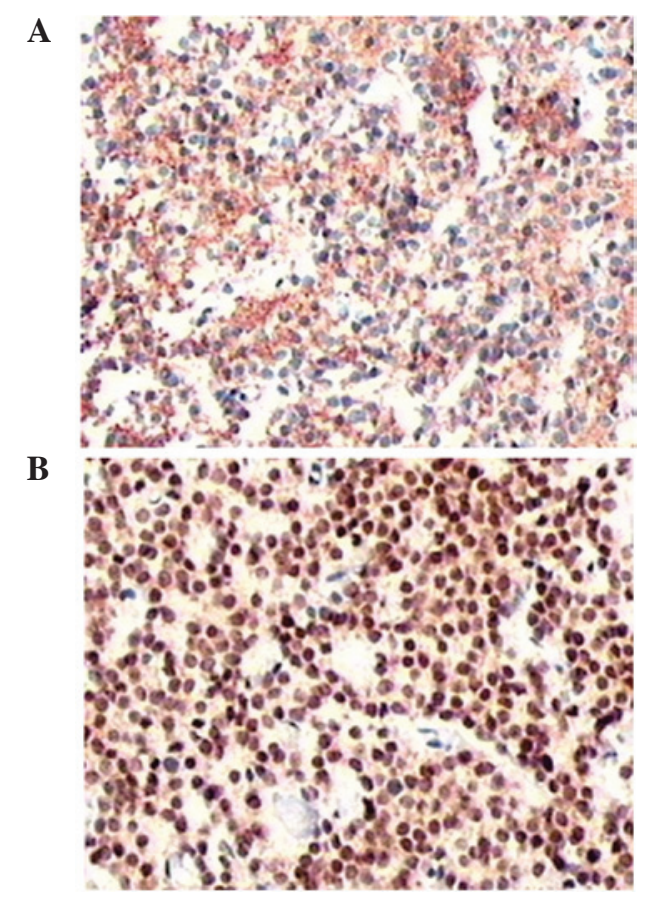

Figure 3. Immunohistochemical staining revealing expression of (A) synaptophysin and (B) neuronal nuclear antigen in the tumor cells. Magnification, x 100 .

and mouse anti-human Ki67 (cat. no. ab8191; 1:50; Abcam) antibodies overnight at $4^{\circ} \mathrm{C}$. The sections were then washed with PBS, incubated with biotinylated goat anti-mouse (cat. no. ab97233; 1:300; Abcam) or goat anti-rabbit (cat. no. ab97188; 1:300; Abcam) secondary antibodies for $30 \mathrm{~min}$ and staining was visualized using an optical microscope (CX23; Olympus Corporation). Immunohistochemical staining revealed that the tumor cells expressed the neuronal differentiation markers, synaptophysin and neuronal nuclear antigen (Fig. 3). Ki-67, a marker for proliferation index, was detected in $10 \%$ of the tumor nuclei. The patient was discharged from hospital without further treatment, including radiotherapy or chemotherapy. The patient was followed up for 3 months post-surgery, with magnetic resonance imaging (MRI; Signa HDe 1.5T; GE Healthcare Bio-Sciences, Pittsburgh, PA, USA) of the brain demonstrating no evidence of tumor recurrence (Fig. 1C). After the initial 3 month followup period, the patient was followed-up every 6 months, and at the time of writing no recurrence had been identified.

\section{Discussion}

CNC is most prevalent in adolescents and young adults, and is often located in the lateral ventricles. In 1997, Giangaspero et al (5) reported the first case of a tumor that mimicked CNC, but was located in an extraventricular region. In 2007, these tumors were classified by the WHO as a novel brain tumor entity (4), and the incidence of EVN remains extremely rare, with Liu et al (6) reporting a worldwide incidence of only $0.13 \%$. The development of CNC appears to have no significant gender predilection; however, the literature indicates that EVN has a slight female predominance and a median patient age of 38 years (range, $5-76$ years) $(7,8)$.

Increased intracranial pressure is the most common clinical presentation, particularly for $\mathrm{CNC}$ of the lateral ventricles. Other symptoms may vary from seizure to gait disturbances depending on the tumor location. The most frequent symptom of EVN is complex partial seizure, followed by headaches, precocious puberty, cranial nerve paralysis, and on rare occasions, hemorrhage (9). Various other non-specific symptoms are also associated with the disease, including headache, dizziness, weakness, and memory and consciousness dysfunction (10). A large number of patients present with these non-specific symptoms, and thus there are no definitive symptoms to diagnose EVN.

EVNs do not present with specific imaging characteristics. On imaging analysis, EVNs may appear as well-circumscribed, round or multilobular, hypodense or isodense masses, which are homogeneously-enhanced with contrast medium, as observed in the present case. 
Liu et al (6) reported that cystic degeneration was observed in 6 out of $9(66 \%)$ EVN cases, with calcification in 34\% and perilesional edema in $44 \%$, whilst Patil et al (11) reported that cystic degeneration was present in $71 \%$ of 9 cases and perifocal edema in 57\%. MRI may exhibit an isointense or slightly hyperintense signal compared with the cortex on T1- and T2-weighted images. The majority of EVN tumors are solid, but largely cystic masses, and mural nodules may be identified. Moderate enhancement is typically observed in these tumors, however, following administration of a contrast agent, mild to strong heterogeneous enhancement may be exhibited (12-14). Common differential diagnoses include oligodendroglioma, ependymoma and dysembryoplastic neuroepithelial tumors, all of which usually lack a clear margin. Contrast enhancement and cystic changes also assist in distinguishing EVNs from other lesions. MR spectroscopy studies of EVNs have demonstrated a prominent choline peak with a notably decreased or absent $\mathrm{N}$-acetylaspartate peak when compared with intraventricular neurocytomas (15).

A characteristic histological feature of EVNs is their composition of monotonous neoplastic cells with round, regular nuclei embedded in a matrix of fine neuronal processes, known as a neuropils. These neuropils are much finer than glial processes and exhibit a 'velvety' appearance, assisting their differentiation from typical rosettes observed in an ependymoma (16). Atypical EVNs are characterized immunohistochemically by a Ki-67 index of $>2 \%$, or by atypical histological features, including increased mitotic activity, focal necrosis and vascular proliferation (17). Histologically, EVN may also resemble oligodendroglioma, therefore, immunochemistry is required to a make an accurate diagnosis. Oligodendrogliomas may exhibit a limited amount of synaptophysin positivity in the tumor cell cytoplasm, but not to the same degree or extent as EVNs $(18,19)$. EVNs may also occasionally carry the $1 \mathrm{p} 19 \mathrm{q}$ codeletion, which is observed in 50-80\% of oligodendroglioma cases (19).

Complete surgical excision is currently considered as the optimal treatment for EVN, but is often limited by tumor proximity to eloquent areas (20). In cases of incomplete resection, available data suggests that radiotherapy offers the same tumor control rates for EVN (11). The success rate of Gamma Knife ${ }^{\circledR}$ radiosurgery has not yet been discussed in the literature due to the limited number of EVN cases.

Post-operative radiation therapy may be effective in reducing tumor size and recurrence rates in cases of aggressive atypical neurocytoma; the recommended dose of $50 \mathrm{~Gy}$ is considered to be the most appropriate for long-term local control in children (20). Additional Gamma Knife stereotactic radiosurgery may be performed post-surgery in order to obtain improved local control and a higher survival rate for cases that cannot be surgically resected (7). The potential benefits of chemotherapy for recurrent tumors and residual lesions is uncertain and is not currently supported in the literature.

In the present case, complete surgical resection was performed due to the clear boundary of the tumor. Further treatment was not necessary, and the patient was followed-up 3 months post-surgery, with MRI of the brain indicating no signs of recurrence.
EVNs are benign in nature, with the exception of aggressive atypical variants (21). There is no association between tumor recurrence and tumor location, and pathology is one important prognostic factor. Atypical EVNs are characterized by a Ki-67 proliferation index of $>2 \%$ or by atypical histological features, including vascular proliferation, focal necrosis and increased mitotic activity. As a result of these features, atypical EVN is difficult to resect completely. Kane et al (22) reported that a typical EVN histology significantly predicts a poor outcome with higher recurrence and mortality rates. Age is an additional prognostic factor, with an age of $>50$ years serving as a risk factor for recurrence, and an age of $<18$ years often associated with atypical features (16).

In conclusion, EVN is extremely rare and presents with varying clinical symptoms and imaging characteristics. The current case described a 49-year-old patient with EVN and discussed the associated clinical presentation, radiological manifestation and histopathological features. Total surgical resection of the tumor was considered as the optimal treatment. However, post-operative radiation therapy may be an effective therapeutic supplement for cases of incomplete resection. In addition, long-term follow-up is essential for cases of atypical EVN.

\section{References}

1. Hassoun J, Gambarelli D, Grisoli F, Pellet W, Salamon G, Pellissier JF and Toga M: Central neurocytoma. An electron-microscopic study of two cases. Acta Neuropathol 56: 151-156, 1982.

2. Sharma MC, Deb P, Sharma S and Sarkar C: Neurocytoma: A comprehensive review. Neurosurg Rev 29: 270-285, 2006.

3. Patel DM, Schmidt RF and Liu JK: Update on the diagnosis, pathogenesis, and treatment strategies for central neurocytoma. J Clin Neurosci 20: 1193-1199, 2013.

4. Louis DN, Ohgaki H, Wiestler OD, Cavenee WK, Burger PC, Jouvet A, Scheithauer BW and Kleihues P: The 2007 WHO classification of tumours of the central nervous system. Acta Neuropathol 114: 97-109, 2007.

5. Giangaspero F, Cenacchi G, Losi L, Cerasoli S, Bisceglia M and Burger PC: Extraventricular neoplasms with neurocytoma features. A clinicopathological study of 11 cases. Am J Surg Pathol 21: 206-212, 1997.

6. Liu K, Wen G, Lv XF, Deng YJ, Deng YJ, Hou GQ, Zhang XL, Han LJ and Ding JL: MR imaging of cerebral extraventricular neurocytoma: A report of 9 cases. AJNR Am J Neuroradiol 34: 541-546, 2013.

7. Choi H, Park SH, Kim DG and Paek SH: Atypical extraventricular neurocytoma. J Korean Neurosurg Soc 50: 381-384, 2011.

8. Choudhari KA, Kaliaperumal C, Jain A, Sarkar C, Soo MY, Rades D and Singh J: Central neurocy toma: A multi-disciplinary review. Br J Neurosurg 23: 585-595, 2009.

9. Taylor CL, Cohen ML and Cohen AR: Neurocytoma presenting with intraparenchymal cerebral hemorrhage. Pediatr Neurosurg 29: 92-95, 1998.

10. Hassoun J, Soylemezoglu F, Gambarelli D, Figarella-Branger D, von Ammon K and Kleihues P: Central neurocytoma: A synopsis of clinical and histological features. Brain Pathol 3: 297-306, 1993.

11. Patil AS, Menon G, Easwer HV and Nair S: Extraventricular neurocytoma, a comprehensive review. Acta Neurochir (Wien) 156: 349-354, 2014.

12. Chen H, Zhou R, Liu J and Tang J: Central neurocytoma. J Clin Neurosci 19: 849-853, 2012.

13. Jaiswal S, Vij M, Rajput D, Mehrotra A, Jaiswal AK, Srivastava AK, Behari S and Krishnani N: A clinicopathological, immunohistochemical and neuroradiological study of eight patients with central neurocytoma. J Clin Neurosci 18: 334-339, 2011. 
14. Yang GF, Wu SY, Zhang LJ, Lu GM, Tian W and Shah K: Imaging findings of extraventricular neurocytoma: Report of 3 cases and review of the literature. AJNR Am J Neuroradiol 30: 581-585, 2009.

15. Ueda F, Suzuki M, Matsui O and Uchiyama N: Automated MR spectroscopy of intra- and extraventricular neurocytomas. Magn Reson Med Sci 6: 75-81, 2007.

16. Brat DJ, Scheithauer BW, Eberhart CG and Burger PC: Extraventricular neurocytomas: Pathologic features and clinical outcome. Am J Surg Pathol 25: 1252-1260, 2001.

17. Rades D, Fehlauer F and Schild SE: Treatment of atypical neurocytomas. Cancer 100: 814-817, 2004.

18. Mut M, Güler-Tezel G, Lopes MB, Bilginer B,Ziyal I and Ozcan OE: Challenging diagnosis: Oligodendroglioma versus extraventricular neurocytoma. Clin Neuropathol 24: 225-229, 2005.
19. Perry A, Scheithauer BW, Macaulay RJ, Raffel C, Roth KA and Kros JM: Oligodendrogliomas with neurocytic differentiation. A report of 4 cases with diagnostic and histogenetic implications. J Neuropathol Exp Neurol 61: 947-955, 2002.

20. Rades D, Schild SE and Fehlauer F: Defining the best available treatment for neurocytomas in children. Cancer 101: 2629-2632, 2004.

21. Rades D, Schild SE and Fehlauer F: Prognostic value of the MIB-1 labeling index for central neurocytomas. Neurology 62: 987-989, 2004.

22. Kane AJ, Sughrue ME, Rutkowski MJ, Aranda D, Mills SA, Lehil M, Fang S and Parsa AT: Atypia predicting prognosis for intracranial extraventricular neurocytomas. J Neurosurg 116: 349-354, 2012. 